\title{
Improvement of the Photonic Bandgap in Three-Dimensional Diamond Lattice Microstructures Obtained by Two-Photon Polymerization
}

\author{
Ran Hee Kim, Jin Sun Park, Hong-Bo Sun, ${ }^{\dagger}$ Satoshi Kawata, ${ }^{\ddagger}$ and Kwang-Sup Lee

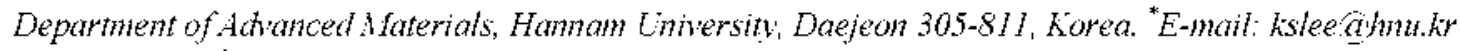 \\ "Department of Electronic Science and Engineering, Jihn Lniversity, China \\ -Department of Applied Physics, Osaka Lniversity, Suita Osaka 565-0871, Japan and JST-CREST, Nanophotonics Laboratory, \\ RIKEN, Hako Saita 351-0198, Japan \\ Received January 9, 2008
}

Key Words : Two-photon polymerization. Photonic bandgap. IR tranșmittance

\begin{abstract}
A photonic crystal $(\mathrm{PhC})$ represents a promising way to control photons in optoelectronic devices. They are periodic structures consisting of photonic band gaps blocking selected frequencies in all directions within the $\mathrm{PhC}$ depending on its lattice parameters. This effect of $\mathrm{PhC}$ on light is similar to the way in which electronic energy is manipulated by semiconductor. ${ }^{l}$ The optical dielectric response has received much attention because of its advantages over the electronic response (i.e.. superior speed and energy -loss characteristics)
\end{abstract}

Since 1989. when Yablonovitch and Gmitter described and demonstrated the existence of a $\mathrm{PBG}^{2}$ many theoretical and experimental studies have been conducted with the aim of finding materials and structures for fabricating $\mathrm{PhCs}$ with a full bandgap. At the beginning. there were two main teclunical issues that had to be resolved to obtain photonic crystals with practical PBGs. One of these issues was the determination of an appropriate cry'stal structure for obtaining a complete photonic bandgap and the other one was the achievement of an adequate fabrication method for producing micro photonic cry stals. Initially. the face-centered cubic (fcc) structure was considered to be the most favorable one for making photonic crystals with an adequate $\mathrm{PBG}^{3}$ Two years later. however. the diamond structure (i.e.. the tetrahedral geometry associated with carbon and silicon crystals) was shown to be the most effective one for realizing a PBG In a microwave band. a complete PBG was successively achieved in a microarray through the spherical voids in a dielectric background ${ }^{+}$The PBG was demonstrated for a tetrahedral diamond structure using a material with a refractive index as small as 1.87 .

Several approaches have been used to fabricate 3D photonic crystals. for example. focused ion-beam etching. "rapid photoprototyping micro-electro-mechanical system (MEMS). ${ }^{6}$ and layer-by-layer projection lithography. ${ }^{7}$ These conventional processes are complex time-consuming and laborious because they have all been extended from twodimensional structures. Alternative approaches. such as selfassembled colloidal PhC. ${ }^{\S}$ multibeam interference techniques." and two-photon absorption photopolymerization (TPP). ${ }^{102}$ : 2 have been competing to achieve good $3 \mathrm{D} \mathrm{PhC}$ structures. TPP has been investigated extensively during the last decade for the fabrication of $3 \mathrm{D}$ micromachines and devices for optical and electrical applications because of the simplicity of patterning through the nonlinear-opticalresponse features. As a consequence. several fcc structuresincluding log piles ${ }^{13}$ and tetrahedral diamond structures ${ }^{14}$ have been fabricated using this technique. and their PBG effects have been examined. We have achieved greater resolutions and smoothness of surfaces in the case of complex 3D structures fabricated by TPP. ${ }^{15}$

The materials used for fabricating 3D structures by TPP must be easily photopolymerizable and transparent at the wavelength of the laser used. In addition, to catch up with the optical characteristics of inorganic and/or semiconductor materials (e.g. the refractive index), nanoparticle-embedded 3D photonic crystals were fabricated and their PBGs were examined using FTIR spectroscopy. Moreover, the introduction of metal oxides was reported to improve the PBG of dielectric structures. ${ }^{16}$ In these studies. polymerizable metalcomplex monomers were used as precursors of the nanoparticles and complex 3D photonic crystals with a confirmed PBG (composed of titanium oxide-polymer and CdS-polymer nanocomposites) were fabricated ${ }^{16.17}$ Here. we report on 3D diamond photonic crystals fabricated by means of TPP using a urethane-acrylate resin doped with copper (II) diarylate monomers. A noticeable PBG improvement (compared to previously reported values) is confirmed by FTIR transmittance spectroscopy

\section{Experimental Section}

Instruments. For fabricating the $\mathrm{PhC}$ stnuctures. a Ti: sapphire mode-locked laser (with an 80 fentosecond pulse width and an $82 \mathrm{MHz}$ repetition rate) was employed. The laser beam was focused with a high-numerical-aperture lens (N.A. $=1.4$. Olympus). Immersion oil was placed between the objective lens and the glass substrate. Displacement of the focal point in the $x, y$ and $z$ directions was detected with an accuracy of less than $1 \mathrm{~nm}$ using a $3 \mathrm{D}$ piezo stage translator. According to the preprogrammed conputer-aided design (CAD) model, 3D diamond ball-stick photonic crystals were obtained on a glass substrate inside a resin drop using the following conditions: $280 \mathrm{~mW}$ laser power. $780 \mathrm{~nm}$ wavelength. and $4 \mathrm{~ms}$ exposure time. The crystals were then washed and immersed in ethanol for two hours to 
remove the resin residue. The detailed conditions are reported elsewhere. ${ }^{1 \hat{3}}$ A scanning electron microscope (SEM. JSM-6400 JEOL) was used to visualize the microphotonic crystals, and the PBG effects were characterized using a Fourier transform infrared spectrometer (FT/RR. ValorIII + Micro 20. JASCO). Thermogravimetric analysis (TGA) diagrants were obtained with a Shimadzı TGA-50 Themogravimetric Analyzer.

TPP Fabrication Conditions. In order to obtain fine PhC structures, we employed the previously reported TPP technjques. which include: (i) the construction of $3 \mathrm{D}$ diamond lattice $\mathrm{PhC}$ arrays in a stick-ball molecular model with a submicron feature size. ${ }^{1 \hat{3}}$ (ii) the pinpoint two-photon writing method ${ }^{1+}$ and (iii) the precompensation TPP technique to prevent shrinkage by increasing the height of the $\mathrm{PhC}{ }^{14}$ The main factors for determining the PBG are the dielectric constant. the refractive-index contrast, and the crystal structure (e.g., the size of the rod or the lattice distance). The wavelength of the PBG center depends on the features of the crystal structure. In the TPP methods. the dimensions of the crystal lattice and the rod size can be principally controlled by adjusting the fabrication conditions. for example, the exposure time and the irradiation power. Preliminary experiments demonstrated that effective lattice constants can be obtained at a laser power of $280 \mathrm{~mW}$. Metal-ion-containing resins were prepared by mixing photopolymerizable urethane-acrylate monomers and oligomers with photoinitiators (SCR-500 resin. Japan Synthetic Rubber. JSR) with copper (II) acrylate monomers (Aldrich). The chemical structure of copper(II) methacrylate and the conceptual two-photon photopolymerization process are portraited in Scheme 1. To improve the PBG effects, $1 \mathrm{wt} \% \mathrm{Cu}^{2+}$ was introduced into the acrylate unit of the oligomer matrix.

\section{Results and Discussion}

3D Diamond Lattice Photonic Crystals. A diamond lattice was adopted to achieve a better PBG of the 3D microstructures. High-quality photonic crystals were successively obtained. Figure $\mathrm{l}(\mathrm{a})$ and $\mathrm{l}(\mathrm{b})$ present detailed electronic

$$
\left(\sum_{0} \mathrm{O}^{-}\right)_{2} \mathrm{Cu}^{2+}+
$$

Copper(II) methacylate<smiles>[R]CC(=C)C(=O)OCC</smiles>

SCR500 resin

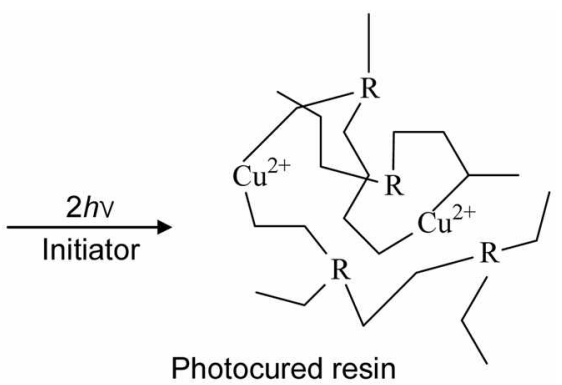

Photocured resin

Scheme 1. Molecular structures of metal complex and the conceptual scheme of the two-photon polymerization with SCR500 resin.

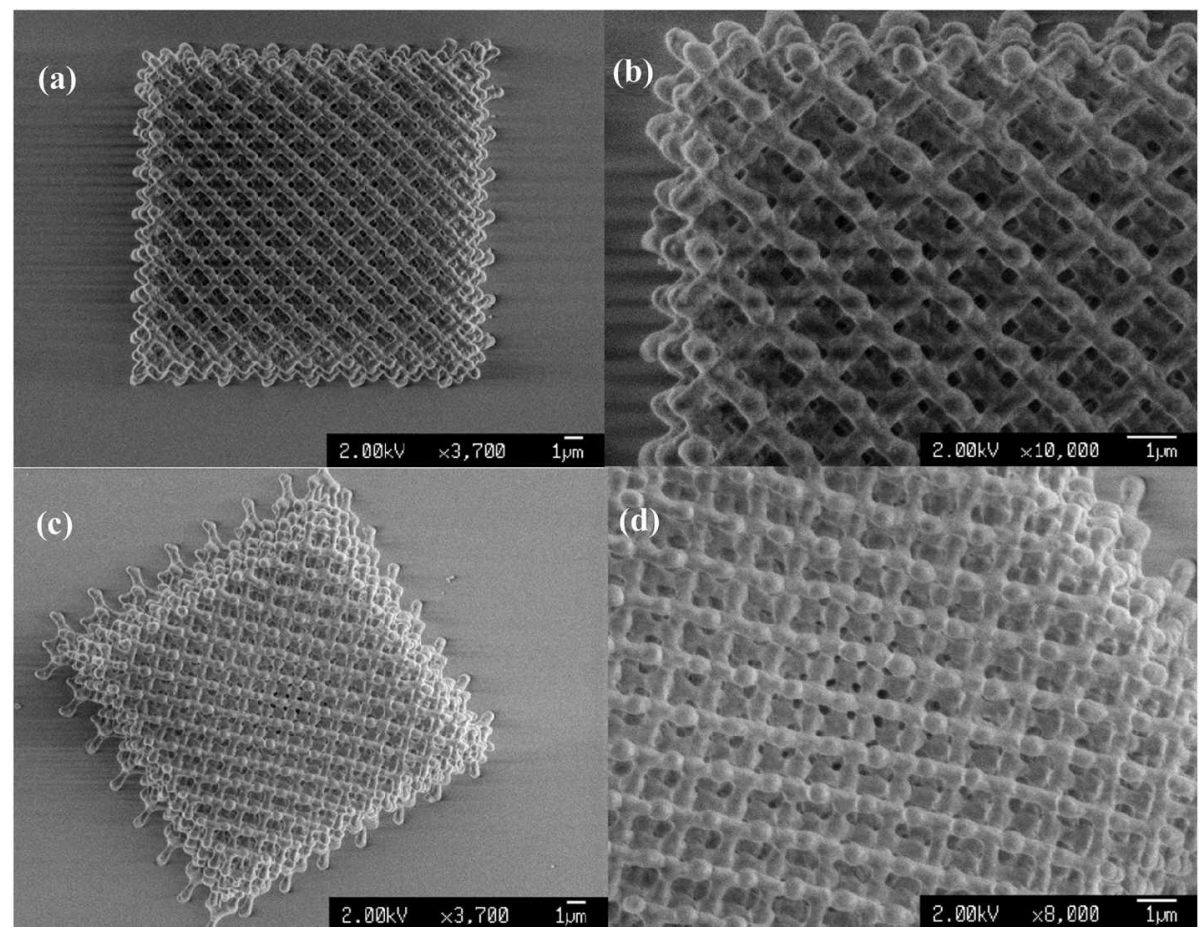

Figure 1. Scanning electron microscopy (SEM) images of two-photon polymerized 3D diamond photonic crystals containing photopolymerizable oligomers doped with $\mathrm{Cu}^{2+}$ : (a) Top view and (b) magnified image of the $8 \times 8 \times 2$ lattice betore heating. (c) Top view and (d) magnified image of the $8 \times 8 \times 5$ lattice after heating. 
photographs of the $\langle 110\rangle$ view of the $8 \times 8 \times 2$ period diamond-crystal repeating unit: in these crystals. the lattice constant is $2.3 \mu \mathrm{m}(\Lambda)$, inplying that the distance between the nearest centers of the lattice atoms is $1.0 \mu \mathrm{m}$. The diameters of the connecting rods and the photonic atoms are 480 and $570 \mathrm{~nm}$, respectively. Heating the polvmeric structures usually caused shrinkage of the polymerized 3D crystals. ${ }^{18}$ After heating (at $210^{\circ} \mathrm{C}$ ) for I hour. the widths of the constructing rods and atoms of an $8 \times 8 \times 5$ period diamond crystal were reduced to 380 and $510 \mathrm{~nm}$ respectively [see Figure I(c) and l(d)]. Furthermore, the top area of the photonic crystal decreased from $18 \times 18$ to $15 \times 15$ $\mu \mathrm{m}$ and the individual rods and atoms exhibited a total shrinkage of about $(20 \pm 3) \%$. Not only a reduction of the crystal dimensions but also a reduction of the crystal lattice parameters was observed (i.e. the lattice constant $\Lambda$ decreased to $1.9 \mathrm{~mm}$ ). It is well established that the lattice distance determines the PBG frequencies of photonic crystals. ${ }^{14}$ Therefore. the heat treatment enables us to adjust the wavelength of the PBGs of dielectric photonic crystalsapart from controlling the laser power and the spacing of the lattice parameters.

Characterization of the Photonic Bandgap. To examine the PBGs of the diamond lattice structures. we performed FTIR transmission measurements in which spin-coated and
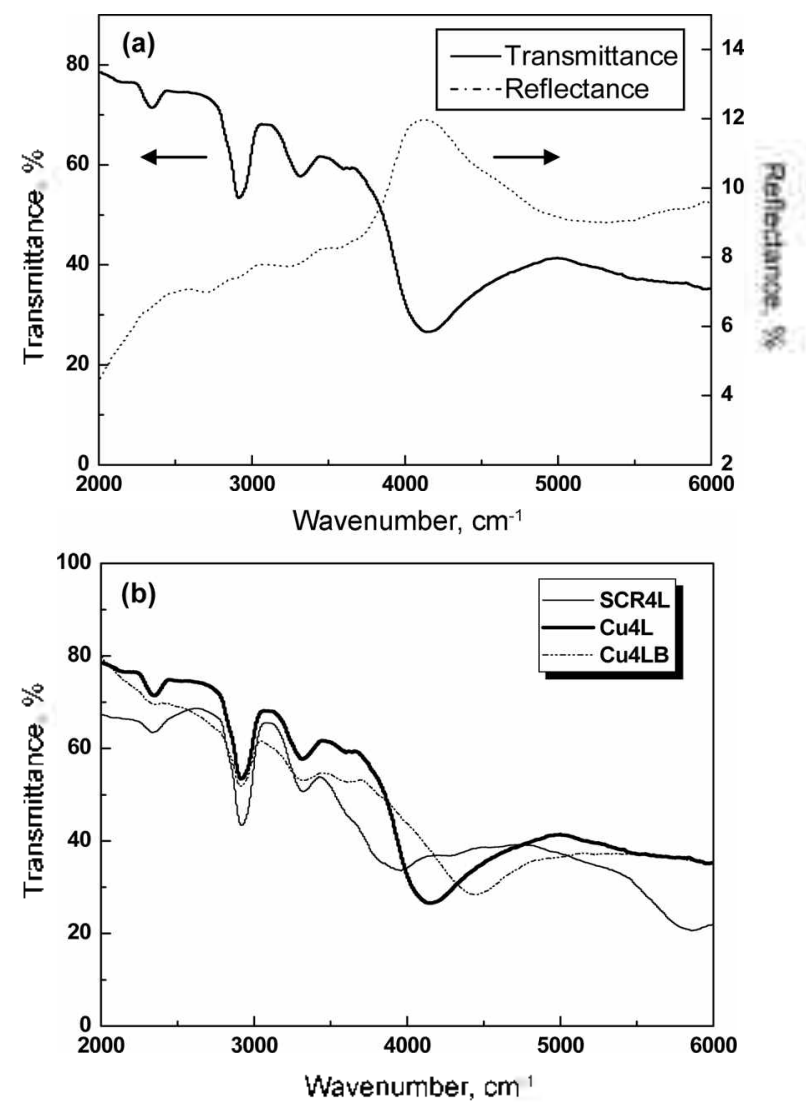

Figure 2. FTIR spectra: (a) transmittance and reflection spectra of $8 \times 8 \times 4$ lattice diamond photonic crystals containing $\mathrm{Cu}^{2-}$, and (b) transmittance spectra of photonic crystals containing $\mathrm{Cu}^{2+}(\mathrm{Cl} 4 \mathrm{~L})$, $\mathrm{Cu} 4 \mathrm{LB}$ atter heating for 1 hour at $210^{\circ} \mathrm{C}$, and the photonic crystal (SCRAL) (the base being UV-cured pure resin).
UV-cured thin films were used as a baseline. $A<100>$ onented $8 \times 8 \times 4$ diamond lattice [Cu4L], constructed by TTP using a metal-ion-containing resm, displayed a PBG at $4100 \mathrm{~cm}^{-1}$ [see Figure 2(a)]. Its reflection spectrum proves that the transmission band corresponds to a PBG thereby being clearly distinguishable from other simple IR absorption bands. A highly efficient PBG (with a $25 \%$ transmittance minimum) was observed at $4100 \mathrm{~cm}^{-1}$. indicating a PBG wavelength of $\lambda=2.4 \mu \mathrm{m}$. This is a remarkable transmittance attenuation compared to those previously reported for diamond and log-pile $\mathrm{PhCs}$ using polymer composites. ${ }^{16.17}$ The nommalized frequency, defined as $\lambda / \Lambda$ (where $\lambda$ is the wavelength of the PBG and $\Lambda$ is the lattice constant), is $1.04 .^{14}$ As shown in Figure 2(b). the IR spectrum of $\mathrm{Cu} 4 \mathrm{~L}$ exlubits an unprovement of $22 \% \mathrm{~m}$ the PBG compared to the value obtaned for the same $\mathrm{PhC}$ structure fabricated using the pure SCR resin (33\% transmission at $3900 \mathrm{~cm}^{-1}$ ). This improvement can be explained by the successful incorporation of copper into the resin. which induces a certain change in the properties of the dielectric material. After heating the $\mathrm{PhC}$ of the metal-containing resin $[\mathrm{Cu} 4 \mathrm{~L}]$ for 1 hour $\left(\right.$ at $210^{\circ} \mathrm{C}$ ), the transmission minimum of $\mathrm{Cu} 4 \mathrm{LB}$ (with an identical $8 \times 8 \times 4$ lattice unit) moved to $4400 \mathrm{~cm}^{-1}$. and the transmittance increased to $38 \%$. Heating the polymerized structure caused a three-dimensional shrinkage of the lattice constants. which corresponds to a bandgap wavelength $(\lambda)$ of $2.2 \mu \mathrm{m}$. However, the transmittance micreased to $38 \%$ (the same behavior was observed when the pure-resin PhC was heated).

To examine the impact of increasing the crystal-unit numbers in the $<001>$ direction on the PBG we fabricated 8 $\times 8 \times 2.8 \times 8 \times 5$, and $8 \times 8 \times 8$ three-dunensional $\mathrm{PhC}$ diamond structures (at a laser power of $300 \mathrm{~mW}$ and an exposure time of $4 \mathrm{~ms}$ ) by using an SCR 500 resin containmig copper-acrylate monomers. As shown in Figure 3. the transmittance attenuation is improved (from $30 \%$ to $14 \%$ ) at $3900 \mathrm{~cm}^{-1}(\lambda=2.6 \mu \mathrm{m})$ by varying the crystal-unit number for the $8 \times 8 \times 2,8 \times 8 \times 5$, and $8 \times 8 \times 8$ BD diamond structures. It is evident that the PBG quality increases as the unit of the diamond crystal grows in the $<001>$ direction.

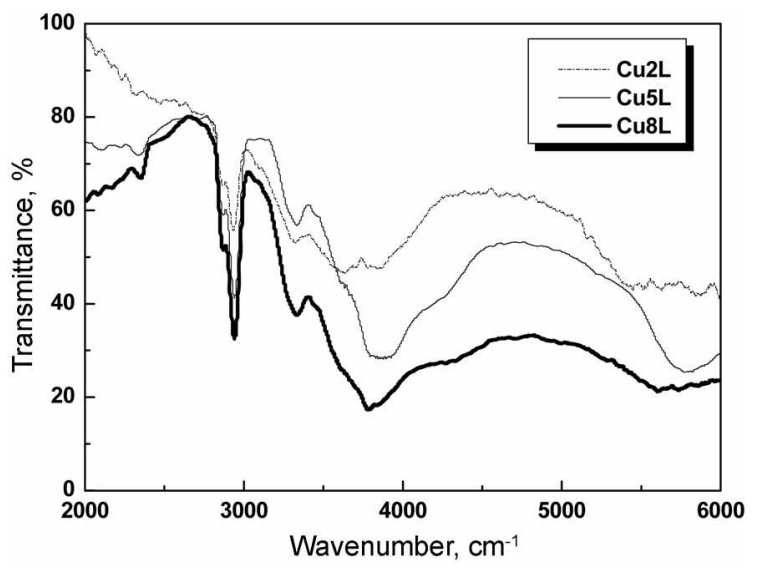

Figure 3. FTIR transmittance spectra of photonic crystals taken after increasing the crystal layer in the $<001\rangle$ direction: $8 \times 8 \times 2$ (CL2L), $8 \times 8 \times 5(\mathrm{CuFL})$, and $8 \times 8 \times 8$ (Cu\&L). 


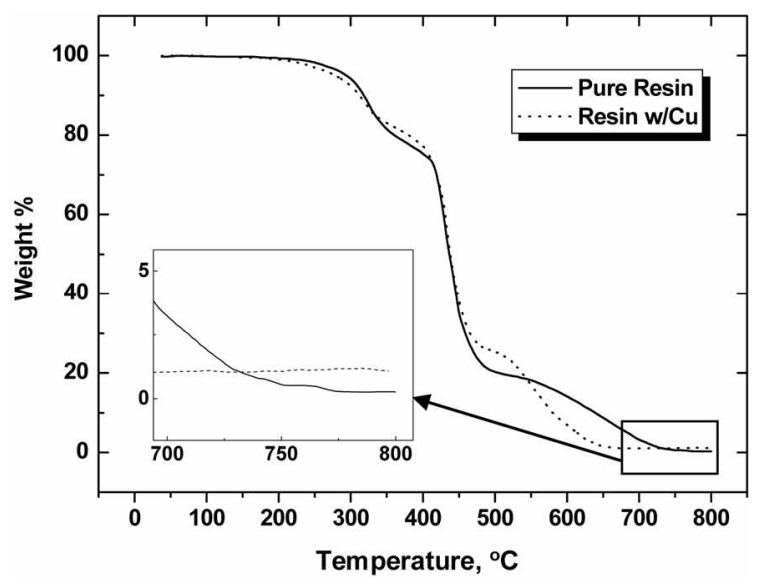

Figure 4 . Thenmal gravimetric diagram of the pure resin (solid line) and of the resin containing Cu(II) (dotted line). The inset shows a magnification of the part of the diagram highlighted in the bos:

The PBG quality also depends on the distance the light covers.

Thermogravimetric Analysis. To further confirm the presence of copper in the dianond structures. we performed a thermogravimetric analysis (TGA) of the solid SCR 500 resin with and without addition of the $\mathrm{Cu}$ acrylate monomer. The temperature was scanned from 30 to $800^{\circ} \mathrm{C}$ (at a rate of $20^{\circ} \mathrm{C} / \mathrm{min}$ ) under nitrogen atmosphere. Figure 4 shows the TGA diagram, and the inset shows a magnification of the part highlighted in the box. Above $500^{\circ} \mathrm{C}$. the degradation behavior of the two crystals exhibited some discrepancy. and at the end of the heating process, a funal weight difference was observed between them. The copper content was found to be $0.78 \mathrm{wt} \%$; despite this low concentration, however, the PBG increase (of $22 \%$ ) was quite noticeable. Further studies at higher copper concentrations and including alternative metal ions are underway in our group.

\section{Conclusions}

Three-dimensional diamond lattice $\mathrm{PhC}$ structures with a high resolution were successively fabricated using TPP. The introduction of a metallic component into the photopolymerizable matrix was shown to improve the PBG effects. A large PBG-effect improvement. with a transmission attenuation to $14 \%$ at $3900 \mathrm{~cm}^{-1}$, was accomplished. However, not only the introduction of a metal component into the photo- polymerizable resin but also the modification of the lattice parameters was shown to enhance the PBG effects of organic $\mathrm{PhC}$ structures. This work provides a useful way to fabricate 3D micro/nanostructures with an improved PBG function for optoelectronic devices and integrated systems

Acknowledgments. This work was supported by the TITKAIST Core University Collaboration Program, sponsored by the Japan Society for the Promotion of Science (JSPS) and the Korea Science and Engineering Foundation (KOSEF). One of us, K.-S. LEE would like to thank the Active Polymer Center for Pattern Integration (ERC R11-2007-05001002-0) and the Asian Office of Aerospace Research and Development (AOARD). USA. for their support.

\section{References}

1. Joatnopoulos. J. D.: Meade. R. D.: Wint1. J. N. Photonic Crnstals: Princeton University Press: Princeton. NJ. USA. 1995.

2. Yablonovitch, E.; Gmitter. T. J. Phys. Rev Lett. 1989, 63. 1950.

3. Ho. K. M.: Chen, C. T.; Soukoulis. C. M. Phys. Rev Lett 1990. 65. 3152 .

4. Yablonovitch. E.: Gmitter. T. J. Phus Rev: Lett. 1991. 67. 2295.

5. Chelnokof. A.: Wang. K.: Rowsont. S.: Garche. P.: Lourtioz. J. M. Appl. Plys. Lett. $2000,77,2943$.

6. Landers, R: Hubner, U.; Schmelzeisen, R: Mulhaupt. R. Biomaterials 2002. 23, +137 .

7. Lit]. S. Y.: Hleming. I. G.: Hetherington. D. L.: Smith. B. K: Biswas. R.: Ho. K. M.: Sigalas. M. M.: Zubrzycki. W.: Kurtz. S. R.: Bur. T. Nature 1998. 394. 251.

8. Yang. S. M.; Miguez. H.; Ozin. G. A. Adv Fuct Hater: 2002. 425. 12.

9. Kawata. S.; Shoji. S.: Sun. H.-B. IEICE Trans. Electron. $2004,87$. C. 378 .

10. Parthenopoulos. D. A.: Retzepis. P. M. Science 1998. 245. 843.

11. Maruo. S.: Nakamura. O.: Kawata. S. Opt. Lett 1997. 22. 132.

12. Cumpston, B. H.: Ananthavel. S. P. Barlow. S.: Dyer, D. L: Ehrlich, J. E.; Erskine, L. L; Heikal, A. A ; Kuebler. S. M.: Lee. I.-Y. S.: McCord-Maughon, D.: Qin. J.: Rockel. H.: Rumi. M.; Wu. X.L.: Marder. S. R.: Perry. J. W. Nature 1999.398.51.

13. Sur. H.-B.: Matsuo. S.: Misawa. H. Appl. Phus Lett. 1999. 74. 786.

14. Kaneko, K; Sun. H.-B.: Duan, X.-M; Kawata. S. Appl. Phys. Lett. 2003, 83. 2091.

15. Sun. H.-B.: Suwa. T.: Takada, K: Zaccaria, R. P.: Kim. M.-S.; Lee. K.-S.: Kawata. S. Appl. Phys Lett. 2004. 85. 3708.

16. Sut. H.-B.: Don. X.-Z.: Nakantishi. S.: Chen. W.-Q.: Duan. X.-M.: Kawata. S. Appl. Phos A 2007. 86.427.

17. Duan. X.-M.: Sun. H.-B.: Kaneko, K.: Kawata. S. Thin Solid Filhs 2004. 453.518.

18. Takada, K: Sun, H.-B.: Kawata, S. Appl. Phys. Lett. 2005, 86. 071122 . 